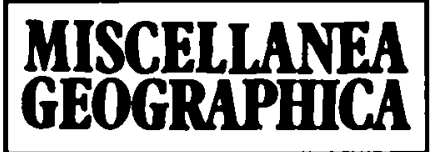

WARSZAWA 1992 Vol. 5

Bohdan Jalowiecki

\title{
INSTITUT EUROPÉEN DU DÉVELOPPEMENT RÉGIONAL ET LOCAL PRÈS L'UNIVERSITÉ DE VARSOVIE
}

Les activités de l'Institut Européen du Développement Régional et Local, aujourd'hui indépendant mais créé et, dans les premiers temps, fonctionnant dans le cadre de la Faculté de Géographie et d'Études Régionales, portent sur la recherche interdisciplinaire régionale et locale.Les groupes d'étude qui réunissent géographes, économistes, sociologues et politologues analysent des problèmes choisis au point de vue de nombreuses disciplines dans le but d'examiner toutes les faces de problèmes étudiés. En 1991, les chercheurs de l'Institut ont réalisé deux programmes de recherche de grande envergure.

Dans le domaine d'études régionales, on a réalisé le programme sur les possibilités et les chances de restructuration des régions polonaises, en vue d'une coopération européenne. L'effort principal des chercheurs s'est concentré sur les problèmes suivants:

- conditions macroéconomiques et macropolitiques de la restructuration des régions;

- analyse de la situation, des conditions et des possibilités de restructuration des trois principaux types de régions, à savoir: région ayant des chances de développement, région moyenne, région qui décline;

- problèmes relatifs à une coopération régionale frontalière;

- analyse des causes de la formation des pôles locaux de la croissance et de la stagnation;

- analyse du rôle et de la place de la Pologne dans les réseaux transeuropéens de l'infrastructure de transports.

Les recherches déjà réalisées ont fourni une certaine somme de connaissances nouvelles sur les régions polonaises dans les conditions de la transformation du système; un accent particulier a été mis sur ces phénomènes qui peuvent devenir importants au point de vue de la dynamique du développement du pays dans le contexte de la coopération européenne. Les thèses principales du Programme peuvent être résumées en quatre points:

1. De nombreux auteurs ont constaté que dans le programme des réformes de la société, de l'économie et de l'État, les références spatiales sont plus que rares. La politique économique de l'État vise, en premier lieu, la réalisation d'objectifs 
macroéconomiques sans prendre en considération les aspects spatiaux du développement économique. Une seule exception en est une recommandation de porter une attention sur les espaces où un chômage régional est en croissance. Il en résulte que les autorités centrales ne réalisent aucune politique régionale, par contre on a admis qu'une telle politique reste dans la compétence des voïvodies voire des communes.

Les voïvodies, pourtant, en tant qu'unités trop petites ne jouissant pas d'une indépendance économique, ne sont pas capables de réaliser la politique régionale. Un autre obstacle est le fait que contrairement à la commune, la voïvodie n'est pas le sujet autonome donc ne peut pas réaliser sa propre politique. A l'échelle de voïvodie, d'un côté les cadres qualifiés font défaut, de l'autre - il n'y a pas de bases méthodologiques suffisantes à mettre au point une politique régionale.

L'État ne poursuit pas une politique régionale, il n'a pas, non plus, des conceptions du développement régional, par contre on parle beaucoup de l'ainsi dite "régionalisation", c'est-à-dire création de plusieurs unités assez grandes, dont le degré d'autonomie serait important. Tout de même, en dehors d'une conception d'autonomisation, nettement idéologique, il n'existe pas d'études de valeur concernant ces problèmes.

2. Comme il en résulte des recherches effectuées dans le cadre du programme, le territoire de la Pologne est de caractère non continu et se divise en espaces distincts dont les caractéristiques économiques et culturelles sont différentes.

Sur la carte de la Pologne, les régions ayant des chances de développement assez grandes se distinguent nettement. Ce sont: la région de Grande-Pologne, l'agglomération varsovienne, et l'agglomération de Gdańsk avec la région d'Elbląg. D'autres régions avec des perspectives de développement: la voïvodie de Bielsk et les régions du Nord-Est du pays, celles-ci vu un assez bon état de l'environnement naturel.

La localisation de nouvelles entreprises confirme ce diagnostic, ces mêmes régions connaissant également la dynamique la plus élevée de la croissance de l'emploi dans le secteur privé.

Les régions qui déclinent sont un autre type d'espace - leur développement passé basait principalement sur l'industrie d'extraction et l'industrie lourde. C'est le cas des voïvodies de Katowice et de Wałbrzych. Ce sont des territoires de la catastrophe écologique, de la mortalité très élevée, des conditions de vie qui ne cessent d'empirer et, dans le proche avenir, du chômage massif.

Parmi les régions qui déclinent se trouve également l'agglomération de Łódź - centre important de l'industrie textile. Dans ce cas, cependant, il existe des possibilités de restructuration, plus prometteuses que dans le cas des régions à l'industrie d'extraction et métallurgique.

Le troisième type, enfin, - la région moyenne - domine dans une grande partie du territoire de la Pologne. Là, nous nous trouvons devant un problème assez intéressant relevant du domaine de la méthodologie de la politique régionale, problème qui n'est pas encore résolu par aucun des pays de la Communauté Européenne. Le problème consiste en ce que les régions aux 
possibilités considérables de la croissance économique continuent de se développer toutes seules, les régions qui déclinent ou les régions arriérées obtiennent des moyens financiers permettant leur restructuration, mais les régions moyennes sont abandonnées à elles-mêmes. Il y a des cas où une telle région réussit à créer une dynamique de développement ce qui lui permet de passer au premier groupe. Il arrive cependant que la stagnation s'approfondit et la région devient ainsi relativement plus arriérée.

3. Dans le territoire de la Pologne, les pôles de la croissance et de la stagnation à l'échelle locale sont bien visibles. Il y a, p.ex., des villes situées côte à côte dont l'une se développe mieux que les autres. L'une des études faisant partie du Programme procède à une comparaison des deux villes, et notamment Dębica et Jarosław, et fournit d'intéressantes hypothèses essayant d'expliquer ce phénomène.

Dębica, ville typiquement industrielle se développant rapidement dès les années trente, après la Seconde Guerre mondiale a été soumise aux processus d'industrialisation "socialiste".

La plupart des habitants de la ville sont venus de la région environnante et leur stage urbain est plutôt court. Les employés des établissements industriels de Dębica sont, pour la plupart, des "ouvriers-agriculteurs". La classe moyenne de même que les traditions bourgeoises n'y existent pas.

De l'autre côté, il y a Jarosław, ville aux traditions historiques, important centre sous-régional. L'étape de l'industrialisation de l'après-guerre ne l'a pas menagée mais c'était une industrialisation organique dans un sens, et qui n'a pas complètement détruit l'ancienne structure de la ville. Jarosław peut se prévaloir d'une classe moyenne et des traditions bourgeoises. Ainsi, contrairement à Dębica, on y note une plus grande dynamique du développement d'entreprises privées et celle des transformations du paysage urbain.

Il en est de même pour le villes de Gdańsk et de Gdynia, cette dernière groupant la plupart des entreprises privées de la région.

Nos recherches semblent confirmer l'hypothèse selon laquelle les traditions sociales et culturelles, mais surtout la présence de la classe moyenne et des traditions bourgeoises, sont aujourd'hui le facteur dynamisant les processus de développement d'entreprises privées. Les centres industriels avec l'ainsi dite classe ouvrière de grande industrie, les villes avec une monoculture industrielle ont beaucoup moins de chances de développement.

4. L'ouverture des frontières à l'Ouest et au Sud, et la semi-ouverture à l'Est met un accent sur la nécessité de développer les régions frontalières. Dans le cadre du Programme, on a effectué des études concernant une coopération des villes situées à la frontière entre la Pologne et l'Allemagne, à savoir Zgorzelec Görlitz, Gubin - Guben, Słubice - Francfort-sur-l'Oder. Ce sont des villes que la frontière divise en deux,villes où, avant la guerre, il existait un seul type d'espace urbain. Après 1945, les parties des villes situées des deux côtés de la frontière se développaient indépendamment. Aujourd'hui, apparaissent les signes précurseurs d'une coopération visant une reconstitution des liens anciens. 
Un autre phénomène caractérisant les régions frontalières non seulement entre la Pologne et l'Allemagne mais observé également entre la France et l'Allemagne ou l'Allemagne et les Pays-Bas sont des échanges commerciaux à l'échelle individuelle. Ces échanges se développent avec un bel dynamisme, surtout du côté polonais de la frontière où de nombreux produits sont moins chers que le mêmes dans les Lands orientaux de la République Fédérale.

Le deuxième Programme de recherches concernait les problèmes relatifs au développement local et se divisait en deux étapes. Les recherches effectuées lors de la première étape portaient sur le fonctionnement de l'autogestion locale, un an après les premières élections libres. Les recherches effectuées lors de la seconde étape étaient consacrées au développement des entreprises à l'échelle locale.

Les recherches sur le fonctionnement de l'autogestion locale ont embrassé plus de 250 communes. Les informations de base ont été fournies par env. 230 maires et présidents des conseils. On a également interviewé tous les conseillers municipaux ou communaux de vingt localités choisis; dans cinq localités, on a interviewé également les habitants.

Nos recherches font partie d'études internationales poursuivies en TchécoSlovaquie, Norvège, Pologne et Hongrie. Qu'il nous soit permis d'espérer que les études ainsi conçues fourniraient des informations de valeur sur le fonctionnement des autogestions territoriales dans de "nouvelles démocraties" et montreraient des caractéristiques du passage du système autoritaire au système démocratique.

Le compte rendu d'études sur le fonctionnement des autogestions locales apporte des caractéristiques individuelles de membres de nouvelles autorités locales - âge, sexe, éducation reçue, et orientation politique. Une remarque d'ordre général s'impose - les élections ayant amené à un échange d'une grande partie d'autorités locales, les organes de l'autogestion sont constitués principalement de membres élus pour la première fois. L'étendue des échanges d'élites locales était plus grande en ville qu'à la campagne où, parfois, il n'y avait pas d'alternative. Dans la plupart des cas, les élites locales sont constituées d'hommes - on y observe également un certain rajeunissement. La majorité de membres des organes de l'autogestion locale semblent éviter les partis politiques et ne déclarent pas leurs options politiques.

Le compte rendu présente ensuite la dépendance entre la structure sociale et les attitudes politiques d'élites locales d'un côté et celles de simples citoyens, de l'autre. On y a caractérisé les scènes politiques locales et les directions des transformations en vue. Sont présentées également les conditons aussi bien intérieures qu'extérieures de la réalisation de la conception de l'autogestion territoriale. Très souvent, les organes de l'autogestion se heurtent contre les difficultés financières qui rendent impossible la réalisation de leurs principales fonctions, à savoir la satisfaction des besoins et aspirations des habitants. Le cadre juridique du fonctionnement de l'autogestion n'est pas définitivement établi, surtout les prin- 
cipes de financement des budgets locaux. Une autre difficulté - espérons que passagère - est l'inexpérience de nouvelles élites locales.

La dernière partie du compte rendu présente la situation financière d'autorités locales, les problèmes locaux et les problèmes économiques qui devraient être résolus dans l'avenir immédiat, les stratégies de développement adoptées par les autorités locales, les attitudes envers les différenciations interrégionales de même que celles à l'égard de la propriété privée, les stratégies de stimulation du développement de l'économie locale, et la politique financière des autorités locales.

Les recherches d'une telle envergure donc très coûteuses n'ont été possibles que grâce à la participation financière du Norwegian Research Council for Applied Social Sciences.

L'Institut Européen du Développement Régional et Local porte également son intérêt sur les problèmes européens dans le large sens de ce mot. Une conférence scientifique ayant pour thème "Europe unifiée - défi 2000 " a été organisée dans le but de faciliter les échanges d'opinions sur l'avenir de l'Europe. Une vingtaine de chercheurs représentant sept pays européens, et notamment Autriche, Belgique, Grèce, Allemagne, Suisse et Pologne ont participé à cette rencontre. Le sujet principal en étaient les problèmes relatifs au développement régional dans la perspective de l'Europe unifiée. Au cours de la conférence on a, entre autres, présenté des problèmes suivants: espace, crise et restructuration, développement et incertitude de l'Europe des régions, Conférence sur la Sécurité et la Coopération en Europe et le rôle de la culture dans l'Europe nouvelle, diaspora dans l'espace européen, colonisation par unification - mauvais exemple pour l'Europe,

La conférence a permis un échange d'opinions entre les chercheurs polonais et ceux venus d'Europe occidentale sur les chances de la Pologne à s'intégrer avec l'Europe dans la perspective de l'an 2000.

Une autre réalisation de l'Institut est une enquête concernant la place de la Pologne en Europe. Puisque les Polonais se trouvent aujourd'hui devant l'un des plus grands défis dans leur histoire, une réflexion intellectuelle sur la situation de la Pologne et les perspectives de son développement dans l'Europe future, réflexion grave et libre d'influences des circonstances actuelles paraît indispensable. C'est ainsi que l'Institut a organisé une enquête conçue à une grande échelle embrassant les intellectuels polonais vivant aussi bien en Pologne qu'à l'étranger. On peut espérer que les résultats de cette enquête apporteraient du nouveau concernant la place de la Pologne en Europe et dans le monde.

En dehors de la recherche scientifique, l'Institut Européen du Développement Régional et Local s'adonne à des activités de promotion de nouvelles initiatives à l'échelle régionale et locale. Il a, entre autres, lancé l'idée de créer des corporations du développement régional. Une conférence a été organisée avec la par- 
ticipation des spécialistes polonais et étrangers, où l'on a discuté des conceptions et des principes de l'organisation et du fonctionnement des corporations du développement régional et local en Pologne avec un accent particulier mis sur les voïvodies d'Elblag, de Białystok, de Łomża, de Suwałki et de Wrocław.

La conférence a mis au point les recommandations suivantes:

- la restructuration des régions devrait s'effectuer par la décentralisation des décisions et de la gestion;

- il est nécessaire de créer un système capable de concurrencer à l'échelle internationale grâce à la recherche des possibilités de développement;

- il est nécessaire de contribuer à la création de l'effet de synergie du secteur public et du secteur privé dans le domaine du développement de la technologie et de l'industrie.

La tâche la plus urgente consiste à formuler les principes du développement régional, à définir le cadre institutionnel conforme aux traits caractérisant la région donnée, à mettre au point un programme de développement pour chaque région, enfin à stimuler les investissements aussi bien polonais qu'étrangers.

Vers la fin de l'année 1990, L'Institut Européen du Développement Régional et Local, en collaboration avec les hebdomadaires Nowoczesnosć (Modernité) et Wspólnota (Communauté) a ouvert le concours ayant pour thème la "Commune 2000 " dont le but principal consistait à inviter les communes à faire l'inventaire de leur potentiel économique respectif afin de mettre au point une offre pouvant attirer des investissements.

Les initiatives du business occidental en Pologne se heurtaient, entre autres, contre le manque d'informations sur les possibilités et les conditions d'investir des capitaux. Les informations d'ordre général ne sont pas suffisantes, le business exige d'informations concrètes permettant d'évaluer d'éventuels bénéfices de même que des risques. L'objectif du concours consistait donc à réunir les informations sur les possibilités économiques des communes particulières et, par là-même, de promouvoir ces dernières sur le marché international. On a décidé que seront recompensées les communes ayant entrepris des initiatives dans le domaine d'investissements, sans attendre l'aide de l'extérieur. Tous les participants sont entrés dans le circuit d'informations international, et les vainqueurs ont été recompensés par une promotion spéciale et une campagne publicitaire organisée à l'aide d'experts mondiaux par l'Union Internationale d'Autorités Locales (IULA) sise à La Haye.

En dehors de la promotion des offres sur le marché international, l'effet principal du concours consiste en l'encouragement à envisager les méthodes modernes du développement local.

En terminant cette brève présentation des activités de l'Institut Européen du Développement Régional et Local, j'aimerais souligner qu'en dehors de l'approche interdisciplinaire dans la recherche scientifique, l'Institut déploie également des activités dans le domaine de la promotion et de l'instruction. 